* Artigo resultante da pesquisa 'Gestão do processo de trabalho na rede de Atenção Básica de saúde em Municípios de Pequeno Porte da região Norte do Paraná', apoiada financeiramente pela Fundação Araucária, por meio da chamada de Projetos 08/2009 Programa de Pesquisa para o SUS: Gestão Compartilhada em Saúde.

1 Universidade Estadual de Londrina (UEL), Programa de Pós-Graduação em Saúde Coletiva - Londrina (PR), Brasil.

alnunes.3@gmail.com

2 Universidade Estadual de Londrina (UEL), Programa de Pós-Graduação em Saúde Coletiva - Londrina (PR), Brasil.

stelamaris08@gmail.com

3 Universidade Estadual de Londrina (UEL), Departamento de Saúde Coletiva - Londrina (PR), Brasil.

brigidagimenez@gmail.com

4 Universidade Estadual de Londrina (UEL), Programa de Pós-Graduação em Saúde Coletiva - Londrina (PR), Brasil.

cordoni@sercomtel.com.br

\section{Força de trabalho em saúde na Atenção Básica em Municípios de Pequeno Porte do Paraná*}

\author{
The workforce in Primary Health Care in Small Sized Counties in \\ Paraná State
}

\author{
Elisabete de Fátima Polo de Almeida Nunes', Stela Maris Lopes Santini2 ${ }^{2}$ Brigida Gimenez \\ Carvalho ${ }^{3}$, Luiz Cordoni Junior ${ }^{4}$
}

\begin{abstract}
RESUMO Este artigo tem como objetivo caracterizar o perfil e as relações laborais dos trabalhadores da Atenção Básica em Municípios de Pequeno Porte localizados no Paraná. Trata-se de estudo transversal descritivo, realizado em 49 municípios do norte do estado, por meio de questionário aplicado aos profissionais de saúde. Os resultados obtidos indicaram a existência de baixa precarização das relações de trabalho, diversidade profissional, escolaridade adequada ou superior ao cargo exercido e vínculos laborais formais. Foram classificados como aspectos negativos a inexistência de plano de carreira, um número insuficiente de médicos, a multiplicidade de vínculos empregatícios e o descumprimento de carga horária. Deste modo, recomenda-se a implantação de políticas específicas aos municípios avaliados, bem como a implantação de medidas de acompanhamento/avaliação.
\end{abstract}

PALAVRAS-CHAVE Recursos humanos em saúde; Gestão em saúde; Força de trabalho; Atenção Primária à Saúde.

\begin{abstract}
This article aims to characterize the profile and the labor relationships of Primary Health Care workers in Small Sized Counties located in Paraná State. It is about descriptive cros$s$-sectional study, applied in 49 counties in northern state, through an applied questionnaire to health professionals. The obtained results have indicated the existence of low precarious in labor relationships, professional diversity, appropriate or superior schooling to the held position and formal employment bonds. Were classified as negative aspects the lack of career plan, an insufficient number of physicians; the multiplicity of employments links, and non-compliance with hourly load. Therefore, it is recommended the implementation of specific policies for the evaluated municipalities, as well as the implementation of monitoring/ evaluation measures.
\end{abstract}

KEYWORDS Human resources in health; Health management; Labor force; Primary Health Care. 


\section{Introdução}

O processo de descentralização dos serviços de saúde no País iniciou-se nos anos 1990, e, atualmente, todos os municípios devem responder minimamente pela gestão e pela execução de ações da Atenção Básica (AB) em seu território. No entanto, decorridas mais de duas décadas desse processo, ainda hoje, nem todos os municípios possuem capacidade técnica e gerencial para responder adequadamente às exigências requeridas por uma gestão tão complexa como é a do trabalho em saúde. Tais considerações se aplicam, especialmente, aos Municípios de Pequeno Porte (MPP), que, segundo o Ministério das Cidades (BRASIL, 2009), têm carência de estrutura para o planejamento e possuem baixa capacidade de gestão urbana para exercer a competência municipal constitucional.

São considerados MPP aqueles que possuem população de até 20.000 habitantes, que representam cerca de $70 \%$ dos municípios do Brasil (IBGE, 2010) e 79,2\% dos municípios do estado do Paraná (PARANÁ, 2013). Apesar da baixa densidade populacional, esses municípios possuem uma alta capilaridade, sendo que, para muitos, a $\mathrm{AB}$ é a única modalidade de assistência à saúde existente.

Com o objetivo de instituir diretrizes nacionais da política de recursos humanos para o Sistema Único de Saúde (SUS), o Ministério da Saúde (MS) criou, em 2003, a Secretaria de Gestão do Trabalho e Educação na Saúde (SGTES), órgão que vem adotando estratégias indutoras para a qualificação da gestão, com investimentos técnicos, políticos e financeiros (PIERANTONI ET AL., 2008).

Apesar dos esforços para estruturar a área de Gestão do Trabalho no SUS, são reconhecidos desafios gerenciais diversificados e cada vez mais complexos, devido às transformações e exigências do trabalho contemporâneo, tais como: escassez de quadros qualificados para o exercício da gestão dos sistemas e serviços, precarização das relações de trabalho, além de várias questões relacionadas à inserção de trabalhadores no serviço, como a distribuição, a fixação, o desempenho profissional, a qualificação, a motivação, a accountability profissional e o resgate de padrões éticos (PIERANTONI ET AL., 2008; PINTO; TEIXEIRA, 2011).

$\mathrm{Na}$ área de gestão do trabalho no SUS, o processo de descentralização para os municípios transferiu para estes decisões e ações antes centralizadas. Nesse processo, os municípios estruturaram uma ampla rede de serviços de saúde no nível da $\mathrm{AB}$ e se tornaram importantes gestores da força de trabalho no SUS.

A gestão do trabalho é um elemento central para a implementação e a consolidação do SUS (PIERANTONI ET AL., 2008), especialmente no espaço da $A B$, em que a prática do cuidado se efetiva por meio da interação entre os trabalhadores e entre estes e os usuários.

Por Gestão do Trabalho no SUS, compreende-se a gestão e a gerência de toda e qualquer relação de trabalho necessária ao funcionamento desse Sistema, e inclui ações de gestão e administração do trabalho, desenvolvimento do trabalhador, saúde ocupacional, além de ações que visem ao controle social nessa área (BRASIL, 2005). Ramos et al. (2009) consideram que

a gestão do trabalho no SUS envolve as questões relacionadas à vida funcional do trabaIhador (ingresso, provimento, movimentação, enquadramento, desenvolvimento na carreira e garantia de direitos trabalhistas), as garantias de condições adequadas de trabalho, participação dos trabalhadores na formulação de políticas (Plano de Cargos, Carreiras e Salários) e na criação de espaços de negociação. (p. 44).

Para os MPP, há o desafio de estruturar a rede de $\mathrm{AB}$, que se caracteriza pelos serviços de tecnologia de baixa densidade, em termos de equipamentos, e de alta 
complexidade nas abordagens preventiva, diagnóstica e terapêutica, isso é, concretizando-se, predominantemente, na dimensão das relações interpessoais. Desta forma, destacam-se como questões estruturantes para o desenvolvimento da rede de $\mathrm{AB}$ a estabilidade e o vínculo de trabalho dos profissionais das equipes de saúde, a capacitação permanente, a possibilidade de negociação das condições de trabalho, a fixação através da existência de plano de carreira, cargos e salários, entre outros aspectos (LACAZ, 2008).

Diante disso, a centralidade do papel dos trabalhadores constitui-se em grande desafio para a implementação da $\mathrm{AB}$, pois é a partir das relações que se estabelecem entre trabalhador-usuário, usuário-trabalhador e trabalhador-trabalhador que ocorre a possibilidade de mudanças e melhorias na prática e no cotidiano dos serviços.

Embora os pequenos municípios representem o maior número, foram identificados poucos estudos que analisassem como a gestão do trabalho tem sido desenvolvida nessas localidades após o processo de descentralização do SUS, principalmente tendo como fonte os próprios trabalhadores. Portanto, conhecer quem são os sujeitos e as relações de trabalho daqueles que atuam na AB dos MPP possibilitará ampliar a capacidade de análise sobre a gestão do trabalho no SUS.

Nesse sentido, este estudo tem como objetivo analisar o perfil e as relações laborais dos trabalhadores que atuam na Atenção Básica do SUS em MPP.

\section{Métodos}

Trata-se de um estudo transversal descritivo, realizado em 49 municípios do Norte do estado do Paraná, com população de até 20.000 habitantes, das áreas de abrangência de três regionais de saúde (RS): $16^{\mathrm{a}} \mathrm{RS}$, de Apucarana, $17^{\mathrm{a}} \mathrm{RS}$, de Londrina, e $18^{\mathrm{a}} \mathrm{RS}$, de Cornélio Procópio. Dos municípios estudados, 16 possuíam até 5.000 habitantes $(32,7 \%), 19$ entre 5.001 e 10.000 (38,8\%), oito com 10.001 a $15.000(16,3 \%)$ e seis de 15.001 a 20.000 habitantes (12,2\%). A somatória da população desses municípios compreendeu 399.189 habitantes (IBGE, 2010).

A população de estudo foi composta pelos trabalhadores que atuavam nos serviços de $\mathrm{AB}$ dos municípios. Foram identificados, inicialmente, pelo Sistema de Cadastro Nacional de Estabelecimentos de Saúde (SCNES), 2.385 trabalhadores. Por ocasião das visitas aos municípios para a coleta dos dados, 383 trabalhadores cadastrados já haviam se desligado das equipes ou estavam atuando em outras áreas que não eram na $\mathrm{AB}$, e por isso foram excluídos; 333 não constavam no SCNES, mas estavam atuando nas equipes da $\mathrm{AB}$, e por isso foram incluídos. Desse modo, a população inicial de estudo foi de 2.335 trabalhadores. Na coleta de dados, houve 470 perdas (trabalhadores em férias, licenças ou que não retornaram o instrumento após três tentativas de contato) e 185 recusas, totalizando 1.680 trabalhadores entrevistados (figura 1). 
Figura 1. População de estudo, identificada mediante consulta ao sistema de cadastro nacional de estabelecimentos de

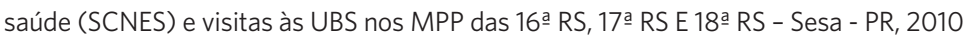

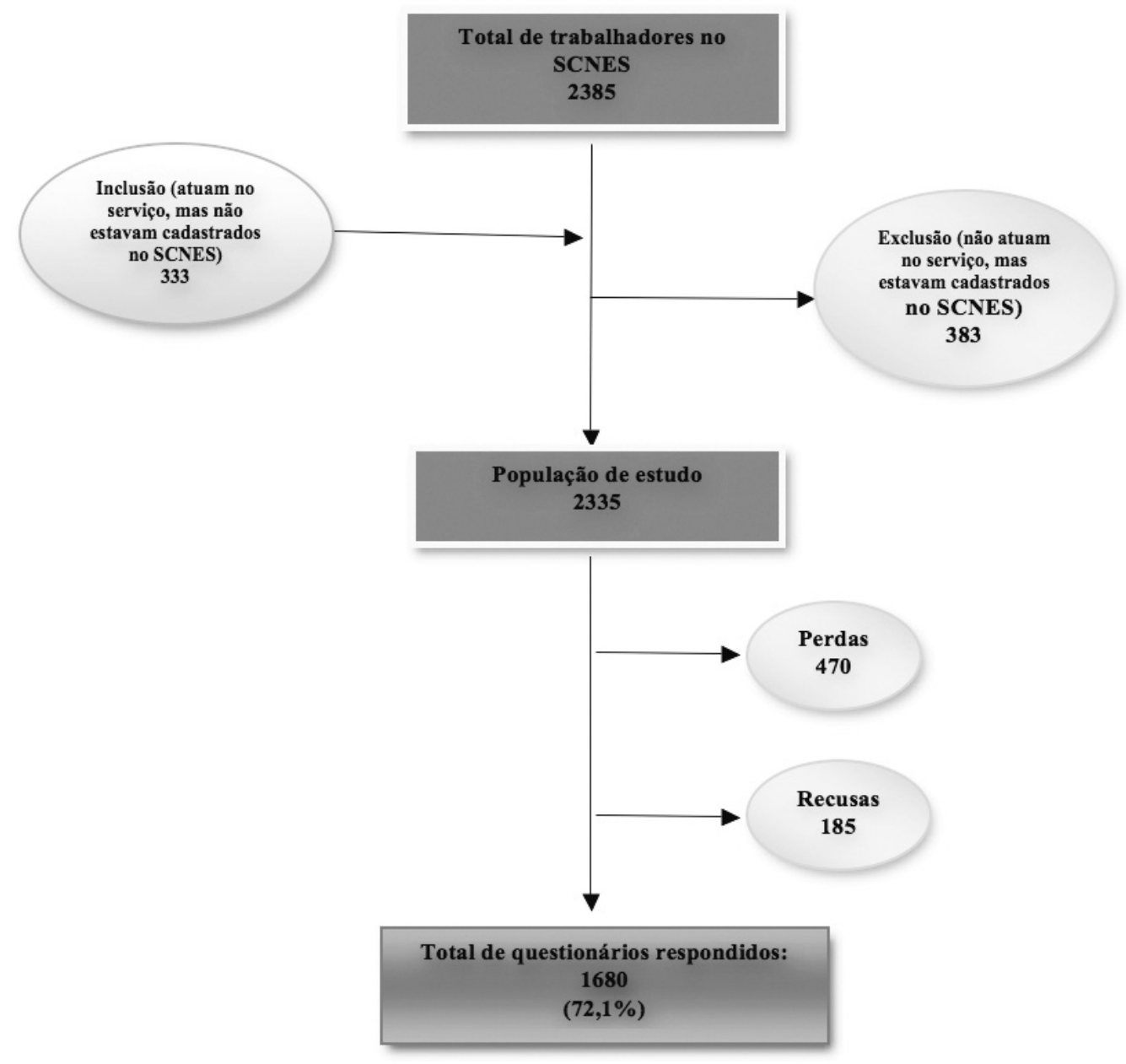

Fonte: Elaboração própria

Os dados foram obtidos no segundo semestre de 2010, por meio de um questionário semiestruturado, aplicado aos trabalhadores de saúde que atuavam nas Unidades Básicas de Saúde (UBS) dos municípios estudados. Os trabalhadores foram abordados em seus locais de trabalho e apresentados aos objetivos do estudo. Os que concordaram em participar preencheram e assinaram o termo de consentimento livre e esclarecido. Para os que não estavam na unidade no dia da coleta, foi deixado o instrumento sob os cuidados da coordenadora da UBS, e devolvido, posteriormente, pelo malote da respectiva Regional de Saúde.

Os dados foram duplamente digitados no Programa EPI INFO, versão 3.5.1. Ao término da digitação, os dois bancos de dados foram confrontados na função comparação dos dados (data compare), permitindo a identificação de campos não concordantes. Após as correções, os dados foram analisados por meio de frequências simples e estudadas as seguintes variáveis: categoria profissional, gênero, faixa etária, formação, remuneração, tempo de atuação no serviço, formas de contratação 
e de vinculação ao trabalho, quantidade de vínculos empregatícios, inserção em um plano de carreiras, cargos e salários e carga horária semanal de trabalho.

Este estudo faz parte da pesquisa 'Gestão do processo de trabalho na rede de Atenção Básica de saúde em MPP da região Norte do Paraná', apoiada financeiramente pela Fundação Araucária, por meio da chamada de Projetos 08/2009 - Programa de Pesquisa para o SUS: Gestão Compartilhada em Saúde.

O projeto foi submetido ao Comitê de Ética em Pesquisa da instituição a que estão vinculados os pesquisadores e aprovado sob parecer $n^{0}$ 232/09, CAAE $n^{0}$ 4255.0.000.268-09.

\section{Resultados}

Ao analisar os dados do SCNES e realizar a adequação dos profissionais que estavam atuando na $A B$ (2.335) no período do estudo, verificou-se uma significativa quantidade e diversidade de categorias profissionais inseridas na $A B$ desses municípios, como se observa na tabela 1.

Nos municípios estudados, verificou-se a relação de 5,85 profissionais de saúde/1.000 habitantes, sendo que, para os médicos, essa distribuição foi de 0,516/1.000 habitantes. Do total de trabalhadores que atuavam na $\mathrm{AB}$, 63,5\% faziam parte das Equipes de Saúde da Família (EqSF), o que correspondeu a uma média de 2,59 EqSF/município.

\begin{tabular}{|c|c|c|}
\hline \multirow{2}{*}{ Categoria Profissional } & \multicolumn{2}{|c|}{ TOTAL } \\
\hline & $\mathbf{N}$ & $\%$ \\
\hline Agentes Comunitários de Saúde & 764 & 32,7 \\
\hline Aux./Téc. Enf. & 386 & 16,5 \\
\hline Médico & 206 & 8,8 \\
\hline Enfermeiro & 173 & 7,4 \\
\hline Agente Controle de Endemias e Téc. Vigilância Sanitária & 167 & 7,2 \\
\hline Dentista & 156 & 6,7 \\
\hline Aux. Saúde Bucal/ Téc. Saúde Bucal & 139 & 6,0 \\
\hline Aux. Adm. & 110 & 4,7 \\
\hline Farmacêutico & 67 & 2,9 \\
\hline Fisioterapeuta & 46 & 2,0 \\
\hline Méd. Vet. & 22 & 0,9 \\
\hline Psicólogo & 20 & 0,8 \\
\hline Nutricionista & 16 & 0,7 \\
\hline Fonoaudiólogo & 13 & 0,6 \\
\hline Ass. Social & 12 & 0,5 \\
\hline Aux. Farmácia & 7 & 0,3 \\
\hline Ed. Físico & 3 & 0,1 \\
\hline Outras & 28 & 1,2 \\
\hline Total & $2335^{\star}$ & 100 \\
\hline
\end{tabular}


Quanto ao tempo de atuação, $69,2 \%$ dos Técnicos de Saúde Bucal (TSB), 40,6\% dos Auxiliares de Enfermagem (Aenf), 40,6\% dos enfermeiros, $40,0 \%$ dos médicos e $38,4 \%$ dos dentistas possuíam de nove a mais de 24 anos de atuação na área, e, do total de trabalhadores entrevistados, 10,5\% possuíam menos de um ano de atuação. Os Agentes Comunitários de Saúde (ACS) eram os profissionais que estavam atuando há menos tempo nas equipes. Destes, $65,8 \%$ atuavam de um a seis anos nos serviços.

Quanto às características sociodemográficas dos profissionais, observou-se predominância de profissionais do sexo feminino em todas as categorias (81,3\%), com exceção da categoria médica, na qual $75,3 \%$ eram do sexo masculino. Nas demais categorias profissionais, verificou-se que mais de 90\% dos Auxiliares de Saúde Bucal (ASB), TSB, enfermeiros, Aenf, e ACS eram mulheres; $88,6 \%$ dos Técnicos de Enfermagem (Tenf) e $58,1 \%$ dos dentistas também eram do sexo feminino.

Quanto às faixas etárias, 76,5\% dos profissionais encontravam-se em idades que variaram de 29 a 44 anos, e a categoria médica foi a única que apresentou maior concentração na faixa etária de 45 a 74 $\operatorname{anos}(52,9 \%)$.

Todos os profissionais possuíam escolaridade adequada ou superior ao exigido. Entre os trabalhadores, destacam-se os Aenf, ASB e ACS, haja vista que mais de $85 \%$ deles possuíam escolaridade acima do exigido para o cargo. Com relação aos ACS, categoria de maior número, verificou-se que $68,4 \%$ haviam concluído o ensino médio, $12,8 \%$ possuíam o ensino superior incompleto e $5,3 \%$ o ensino superior completo. Ainda com relação a essa categoria, $78,9 \%$ já haviam concluído a formação inicial de ACS, com carga horária de 440 horas. Entre as categorias de profissionais com graduação superior obrigatória, $58,1 \%$ dos médicos, $74,8 \%$ dos enfermeiros e $58,8 \%$ dos dentistas eram pós-graduados, sendo que, em nível de mestrado, foram identificados quatro dentistas, um enfermeiro, três médicos e um farmacêutico; nenhum profissional referiu nível de doutorado.

Houve predominância de seleção por concurso público ou teste seletivo, em que $79,3 \%$ de todos os profissionais referiram ter sido assim selecionados. Apesar dessa predominância, foi possível verificar que, aproximadamente, $20 \%$ dos profissionais foram selecionados por outros meios, como análise de currículo, entrevistas, indicações e outros.

As prefeituras foram responsáveis, diretamente, por $83,6 \%$ das admissões dos profissionais, sendo que $77,2 \%$ deles possuíam vínculos empregatícios formais, ou seja, Estatuto do Servidor Público (32,7\%), ou foram contratados via Consolidação das Leis Trabalhistas (CLT) (44,5\%); 4,1\% por contratos temporários e 2,3\% por cargos comissionados. Em contrapartida, 12,4\% dos profissionais foram admitidos por meio de terceirizações. Os demais trabalhadores não informaram a forma de contratação (tabela 2). 
Tabela 2. Modalidade de seleção, tipo de vínculo, tempo de serviço e remuneração dos profissionais das e Equipes de Atenção Básica dos MPP da

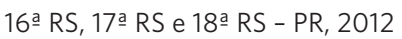

\begin{tabular}{|c|c|c|c|c|c|c|c|c|c|c|}
\hline CATEGORIA PROFIS. & ACS & AENF & ASB & TENF & TSB & Enf. & Dent. & Méd. & $\mathrm{OCP}\left({ }^{\star}\right)$ & Total \\
\hline \multicolumn{11}{|c|}{ Modalidade de seleção } \\
\hline $\begin{array}{l}\text { Concurso } \\
\text { Teste selet. }\end{array}$ & 534 & 160 & 58 & 66 & 23 & 104 & 73 & 53 & 261 & 1332 \\
\hline Currículo & 10 & 1 & & 2 & & 5 & 2 & 7 & 24 & 51 \\
\hline Entrevista & 16 & 7 & 1 & 3 & 1 & 5 & & 7 & 7 & 47 \\
\hline Indicação & 78 & 19 & 7 & 6 & 1 & 26 & 6 & 10 & 51 & 204 \\
\hline Outros & 3 & 2 & & 1 & & 3 & 3 & 8 & 14 & 34 \\
\hline Não resp. & 2 & 1 & & 1 & 1 & & 2 & - & 5 & 12 \\
\hline \multicolumn{11}{|c|}{ Tipo de vínculo } \\
\hline CLT & 380 & 78 & 26 & 20 & 6 & 64 & 44 & 27 & 103 & 748 \\
\hline Estatuto & 123 & 89 & 28 & 42 & 17 & 41 & 31 & 16 & 163 & 550 \\
\hline Cargo Comis & 1 & 1 & 01 & 2 & - & 7 & 1 & 1 & 25 & 39 \\
\hline Cont.Temp. & 15 & 5 & 01 & 4 & - & 6 & 3 & 16 & 18 & 68 \\
\hline Terceiriz. & 117 & 8 & 07 & 10 & 3 & 19 & 2 & 12 & 30 & 208 \\
\hline Outros $\left({ }^{\star \star}\right)$ & - & 1 & - & - & - & 3 & 1 & 1 & - & 6 \\
\hline Não Resp. & 7 & 8 & 3 & 1 & - & 3 & 4 & 12 & 23 & 61 \\
\hline \multicolumn{11}{|c|}{ Tempo de serviço (anos) } \\
\hline$<1$ & 75 & 12 & 2 & 11 & 2 & 20 & 7 & 8 & 40 & 177 \\
\hline $1-3$ & 201 & 19 & 8 & 15 & 2 & 34 & 15 & 9 & 73 & 376 \\
\hline$>3-6$ & 222 & 28 & 19 & 10 & 2 & 29 & 18 & 13 & 45 & 386 \\
\hline$>6-9$ & 72 & 24 & 7 & 9 & 2 & 28 & 5 & 6 & 49 & 202 \\
\hline$>9-12$ & 40 & 24 & 6 & 4 & 2 & 16 & 9 & 8 & 32 & 141 \\
\hline$>12-24$ & 4 & 53 & 14 & 18 & 16 & 8 & 24 & 26 & 53 & 216 \\
\hline Não Resp. & 29 & 30 & 10 & 12 & - & 8 & 8 & 15 & 70 & 182 \\
\hline \multicolumn{11}{|c|}{ Remuneração (em salários mínimos) } \\
\hline$<1$ & 24 & - & - & - & - & 1 & - & - & 4 & 29 \\
\hline 01 & 213 & 25 & 19 & 9 & 4 & - & - & - & 58 & 328 \\
\hline$>1-2$ & 387 & 130 & 38 & 61 & 19 & 5 & 17 & - & 169 & 826 \\
\hline$>2-6$ & - & 10 & - & 2 & 3 & 119 & 57 & 13 & 89 & 293 \\
\hline$>6-10$ & - & - & - & - & - & - & 3 & 31 & 4 & 38 \\
\hline$>10$ & - & - & - & - & - & - & - & 19 & - & 19 \\
\hline Não Resp. & 19 & 25 & 9 & 7 & - & 18 & 9 & 22 & 38 & 147 \\
\hline Total & 643 & 190 & 66 & 79 & 26 & 143 & 86 & 85 & 362 & 1680 \\
\hline
\end{tabular}

Fonte: Elaboração própria

*OCP: Outras Categorias Profissionais

${ }^{\star *}$ Outros: contrato verbal, clínica, por número de consultas, autônomos, cedido pela Sesa, contratado como profissional de nível médio, mas atua como profissional de nível superior, Recibo de Pagamento a Autônomo (RPA)

Tomou-se como base o salário mínimo federal da época da aplicação dos questionários (R\$510,00)

A média salarial, calculada em Salários Mínimos (SM), referida pelos profissionais, foi de 11 SM para os médicos; 3,4 SM para os enfermeiros; 3,3 SM para os dentistas; 1,6 SM para os TSB; 1,4 SM para os Aenf e Tenf; e 1,1 para os ASB e ACS. O salário mínimo federal à época da coleta dos dados era de $\mathrm{R} \$ 510,00$.

Dos profissionais pesquisados, $79,5 \%$ responderam não estar incluídos em Plano de Carreiras, Cargos e Salários (PCCS), sendo essa situação predominante em todas as categorias. 
Quanto ao número de vínculos de trabalho, $78,8 \%$ de todos profissionais referiram atuar em um único vínculo, ou seja, somente na $\mathrm{AB}$ daquele município. No entanto, $75,3 \%$ dos médicos referiram possuir de dois a mais de quatro postos de trabalho, e $61,7 \%$ dos dentistas de dois a três.

Quanto à carga horária semanal contratual, $84,6 \%$ dos profissionais referiram ter sido contratados para uma jornada semanal de 30 ou 40 horas. No entanto, um percentual menor de trabalhadores referiu cumprir tal carga horária $(80,4 \%)$. Ainda no tocante à carga horária contratual, os profissionais médicos e dentistas se distinguem dos demais, tendo em vista que $28,2 \%$ dos médicos e $44,3 \%$ dos dentistas referiram ter sido contratados para uma jornada de 10 a 20 horas semanais (tabela 3 ).

Tabela 3. Carga horária semanal contratada e cumprida na unidade e no total dos profissionais das Equipes de AB dos MPP da 16르 RS, 17a RS e 18ㄹ $\mathrm{RS}$ - PR, 2012

\begin{tabular}{|c|c|c|c|c|c|c|c|c|c|c|c|c|c|}
\hline \multirow{2}{*}{ Profiss. } & \multirow{2}{*}{\multicolumn{2}{|c|}{$\begin{array}{l}\text { 04-10 } \\
\text { № \% }\end{array}$}} & \multicolumn{2}{|c|}{$>10-20$} & \multicolumn{2}{|c|}{$>20-30$} & \multicolumn{2}{|c|}{$>30-40$} & \multicolumn{2}{|c|}{$>40-60$} & \multicolumn{2}{|c|}{ Não Resp. } & \multirow{2}{*}{$\begin{array}{r}\text { Total } \\
\text { № }\end{array}$} \\
\hline & & & № & $\%$ & $\mathbf{N}$ & $\%$ & № & $\%$ & № & $\%$ & № & $\%$ & \\
\hline C.H. semanal Contratada & & & & & \multicolumn{2}{|c|}{ Categoria } & & & & & & & \\
\hline ACS & 3 & 0,5 & - & - & - & - & 634 & 98,6 & 2 & 0,3 & 4 & 0,6 & 643 \\
\hline Aenf & 4 & 2,2 & 1 & 0,5 & 16 & 7,6 & 165 & 87,6 & 2 & 1,6 & 2 & 0,5 & 190 \\
\hline ASB & - & - & - & - & 1 & 1,6 & 63 & 95,2 & 1 & 1,6 & 1 & 1,6 & 66 \\
\hline Dentista & 2 & 2,3 & 39 & 44,3 & 3 & 3,4 & 40 & 47,7 & - & - & 2 & 2,3 & 86 \\
\hline Enfermeiro & - & - & 8 & 6,0 & 4 & 3,0 & 129 & 89,5 & - & - & 2 & 1,5 & 143 \\
\hline Médico & 9 & 10,6 & 24 & 28,2 & 9 & 10,6 & 34 & 40,0 & 4 & 4,7 & 5 & 5,9 & 85 \\
\hline OCP & 7 & 1,9 & 56 & 15,6 & 21 & 5,8 & 267 & 73,4 & 5 & 1,4 & 6 & 1,9 & 362 \\
\hline Tenf & - & - & - & - & 9 & 12,1 & 67 & 83,1 & 2 & 2,4 & 1 & 2,4 & 79 \\
\hline TSB & - & - & 1 & 3,8 & 1 & 3,8 & 24 & 92,4 & - & - & - & - & 26 \\
\hline Total & 25 & 1,5 & 129 & 7,7 & 64 & 3,8 & 1423 & 84,6 & 16 & 1,0 & 23 & 1,4 & 1680 \\
\hline \multicolumn{14}{|l|}{$\begin{array}{l}\text { C.H. semanal cumprida } \\
\text { na unidade }\end{array}$} \\
\hline ACS & 02 & 0,3 & 01 & 0,2 & 01 & 0,2 & 627 & 97,5 & 04 & 0,6 & 08 & 1,2 & 643 \\
\hline Aenf & 06 & 3,2 & 01 & 0,5 & 24 & 12,6 & 147 & 77,4 & 10 & 5,3 & 02 & 1,0 & 190 \\
\hline ASB & 02 & 3,0 & 02 & 3,0 & 03 & 4,6 & 56 & 84,9 & 02 & 3,0 & 01 & 1,5 & 66 \\
\hline Dentista & 08 & 9,3 & 40 & 46,5 & 06 & 7,0 & 29 & 33,7 & - & - & 03 & 3,5 & 86 \\
\hline Enfermeiro & 03 & 2,1 & 08 & 5,6 & 04 & 2,8 & 125 & 87,4 & 01 & 0,7 & 02 & 1,4 & 143 \\
\hline Médico & 12 & 14,1 & 23 & 27,1 & 11 & 12,9 & 29 & 34,1 & 05 & 5,9 & 05 & 5,9 & 85 \\
\hline OCP & 11 & 3,1 & 62 & 17,1 & 21 & 5,8 & 252 & 69,6 & 03 & 0,8 & 13 & 3,6 & 362 \\
\hline Tenf & 01 & 1,3 & - & - & 10 & 12,6 & 64 & 81,0 & 04 & 5,1 & & - & 79 \\
\hline TSB & & & 01 & 3,8 & 02 & 7,7 & 21 & 80,8 & 02 & 7,7 & & - & 26 \\
\hline Total & 45 & 2,7 & 138 & 8,2 & 82 & 4,9 & 1350 & 80,4 & 31 & 1,8 & 34 & 2,0 & 1680 \\
\hline \multicolumn{14}{|l|}{ C.H. semanal Total } \\
\hline ACS & - & - & 01 & 0,2 & - & - & 591 & 91,9 & 26 & 4,0 & 25 & 3,9 & 643 \\
\hline Aenf & - & - & 01 & 0,5 & 19 & 10,0 & 127 & 66,8 & 25 & 13,2 & 18 & 9,5 & 190 \\
\hline ASB & - & - & - & - & 03 & 4,5 & 54 & 81,8 & 02 & 3,1 & 07 & 10,6 & 66 \\
\hline Dentista & 02 & 2,3 & 13 & 15,1 & 03 & 3,5 & 30 & 34,9 & 31 & 36,1 & 07 & 8,1 & 86 \\
\hline Enfermeiro & - & - & 07 & 4,9 & 02 & 1,4 & 115 & 80,4 & 12 & 8,4 & 07 & 4,9 & 143 \\
\hline Médico & 02 & 2,4 & 04 & 4,7 & 04 & 4,7 & 25 & 29,4 & 46 & 54,1 & 04 & 4,7 & 85 \\
\hline OCP & - & - & 13 & 3,6 & 21 & 5,8 & 258 & 71,3 & 32 & 8,8 & 38 & 10,5 & 362 \\
\hline Tenf & - & - & - & - & 05 & 6,3 & 57 & 72,2 & 12 & 15,2 & 05 & 6,3 & 79 \\
\hline TSB & - & - & - & - & 01 & 3,8 & 21 & 80,8 & 03 & 11,6 & 01 & 3,8 & 26 \\
\hline Total & 04 & 0,2 & 39 & 2,3 & 58 & 3,5 & 1278 & 76,1 & 189 & 11,3 & 112 & 6,6 & 1680 \\
\hline
\end{tabular}

Fonte: Elaboração própria 
Quanto à carga horária semanal total de trabalho, considerando todos os vínculos, $76,1 \%$ referiram cumprir jornada semanal de 30 a 40 horas. Novamente, os profissionais médicos e dentistas apresentaram resultado diferente dos demais, sendo que $54,1 \%$ dos médicos referiram cumprir de 40 a 60 horas semanais, e $71 \%$ dos dentistas de 30 a 60 horas semanais.

\section{Discussão}

A presença de equipes multiprofissionais foi bastante marcante. Além dos profissionais das equipes mínimas da Estratégia Saúde da Família (ESF), foram identificados fisioterapeutas, médicos veterinários, psicólogos, nutricionistas, fonoaudiólogos, assistentes sociais, auxiliares de farmácia, educadores físicos. Esses profissionais têm potencial para exercer e apoiar ações de promoção, proteção e vigilância à saúde. Assim, a diversidade de categorias profissionais sugere, ainda que incipientemente, a perspectiva de ampliar a abrangência e o escopo das ações da $A B$, bem como o aumento de sua resolutividade.

No cômputo de todos os municípios, a média de EqSF/município foi de 2,59, o que corresponde, em média, a 3.143 pessoas por equipe. Com base na recomendação do Ministério da Saúde (BRASIL, 2012, P.55), que considera que "Cada Equipe de Saúde da Família deve ser responsável por, no mínimo, 4.000 pessoas, sendo a média recomendada de $3.000[\ldots .$.$] ", pode-se afirmar que a cobertura$ das EqSF está dentro do padrão recomendado pelo MS ou próximo a ele. Tal dado, associado ao elevado percentual de trabalhadores que participavam das EqSF demonstra a grande inserção e a importância da ESF nos municípios avaliados.

Estima-se que, pelo menos, 1,3 bilhão de pessoas no mundo estejam sem acesso aos cuidados de saúde mais básicos, abaixo do percentual recomendado pela Joint Learning
Iniciative, que é de 2,5 trabalhadores de saúde/1.000 habitantes, limite mínimo necessário para atingir a cobertura de saúde adequada (APUD PIERANTONI ET AL., 2008). Nos municípios estudados, verificou-se a relação de 5,85 profissionais de saúde/1.000 habitantes, ou seja, acima dos padrões referidos.

Ao se considerar o parâmetro ideal de um médico por 1.000 habitantes, segundo a Organização Mundial de Saúde (OMS) (CAMPOS; MACHADO; GIRARDI, 2009), constata-se que a região estudada encontra-se abaixo do modelo desejado, pois o coeficiente encontrado foi de 0,516 médico/1.000 habitantes. No entanto, há de se considerar que neste estudo não foram computados profissionais atuantes em outros níveis de atenção, como hospitais, focando somente na AB. Mesmo assim, a proporção médicos/habitantes não deve se alterar significativamente, porque, na maioria dos MPP, os únicos serviços de saúde existentes são os da $\mathrm{AB}$.

Segundo Carvalho, Santos, e Campos (2013), o planejamento da força de trabalho em saúde é um ato político e está condicionado não só ao conhecimento técnico, mas, também, entre outros fatores, ao modelo vigente de atenção à saúde, pois o número, o perfil e a distribuição de trabalhadores da saúde estão articulados a escolhas políticas e a valores e princípios a serem garantidos na organização dos sistemas de saúde. Referem que a razão médicos/habitantes, no Brasil, é o dobro da recomendada pela Organização Mundial de Saúde, e questionam sobre o que explicaria o fato de a cobertura das EqSF ser maior nas áreas onde há menos médicos disponíveis (CARVALHO; SANTOS, CAMPOS, 2013). Embora haja médicos suficientes no País, a fixação dos mesmos em MPP é um dos grandes desafios para a construção do SUS.

Estudando-se as características sóciodemográficas dos profissionais das equipes da $A B$, observou-se predominância de profissionais do sexo feminino em todas as categorias, com exceção da médica, na qual a maioria era do sexo masculino. 
Segundo Wermelinger et al. (2010), desde os anos 2000, já era predominante a força de trabalho feminina na saúde, pois, entre os médicos, as profissionais do sexo feminino representavam $35,94 \%$, e, entre os enfermeiros, $90,39 \%$. Entre os profissionais de nível técnico e auxiliar, a feminização também era expressiva, alcançando $86,93 \%$ dos técnicos e auxiliares de enfermagem.

A fixação dos profissionais na $\mathrm{AB}$ se constitui num desafio para a gestão do sistema. Também, sobre esse aspecto, este estudo sinaliza bons resultados, pois verificou-se que somente $10,5 \%$ dos profissionais atuavam há menos de um ano na $\mathrm{AB}$ desses municípios, $22,4 \%$ trabalhavam de um até três anos e $56,3 \%$ há mais de três anos. Os dados sugerem uma baixa rotatividade de profissionais em MPP e, possivelmente, um maior vínculo com a população. Pode-se inferir, também, que a realização de concursos públicos e a admissão por vínculos estáveis se constituam em estratégias potentes para a fixação desses profissionais.

No entanto, é necessário maior aprofundamento sobre os fatores determinantes da fixação e da rotatividade dos profissionais nos serviços de $\mathrm{AB}$ em MPP, tendo em vista a sua importância para a qualificação da gestão, visando, principalmente, às melhores formas de planejamento, seleção e fixação de profissionais. Tais questões se justificam tendo em vista que a fixação de equipe qualificada está intrinsecamente relacionada à cobertura e à resolubilidade dos serviços, pois, segundo Scherer et al. (2009, P. 723), "A construção do coletivo depende da presença de um mínimo de estabilidade e de certa permanência na organização, pois a confiança e a cooperação se constroem com o tempo".

Ao analisar a escolaridade dos profissionais, verificou-se que os ACS deste estudo apresentaram escolaridade acima da exigida para o cargo, condição que representa um grande avanço se comparada à época da implantação do Programa de Agentes Comunitários de Saúde (Pacs) no Brasil, datada de 1991, quando a condição exigida era a de que soubessem ler e escrever. Mais recentemente, a Lei $\mathrm{n}^{0} 11.350$, de 2006, explicitou, em seu artigo $6^{\circ}$, que a conclusão do ensino fundamental e do curso de qualificação básica para formação de ACS são requisitos para o exercício da atividade (BRASIL, 2006). Quanto à formação inicial para ACS, verificou-se que $21,1 \%$ dos ACS da área em estudo necessitam dessa formação, tanto para atendimento da legislação como para oferecer melhor qualificação e melhores condições para o trabalho.

Há de se destacar que não só os ACS, mas a grande maioria dos profissionais das equipes possuía condições de escolaridade adequadas ou superiores às exigidas para o cargo. Os resultados demonstram que o fato dos profissionais atuarem em MPP, no interior do estado, não os impede de ter acesso a processos de formação e qualificação profissional.

Segundo Lacaz (2008), a educação e a formação de pessoal são formas de valorização da força de trabalho e, por consequência, um instrumento importante de gestão do trabalho. Com referência ao mesmo tema, Mendonça et al. (2010, P. 2357) comentam que

na área da saúde, os avanços da ciência e o desenvolvimento tecnológico acelerado, característicos do modo de produção atual, implicam a necessidade de uma constante atualização de seus profissionais e uma base de conhecimentos interdisciplinar sólida que Ihes permitam enfrentar a diversidade e a 'adversidade' do processo de saúde no mundo atual.

Quanto à admissão e à manutenção dos trabalhadores, importantes resultados foram verificados neste estudo, pois as prefeituras foram diretamente responsáveis pela seleção e pela contratação de $83,6 \%$ do total dos profissionais de saúde que atuavam na $\mathrm{AB}$ dos municípios em estudo, sendo que $77,2 \%$ possuíam vínculos formais com os municípios. 
A partir da década de 1990, passou a existir um mosaico de situações jurídicas e profissionais entre os trabalhadores de saúde, gerando novas formas de gestão do trabalho, como o aumento do número de profissionais contratados como autônomos, terceirizados, incentivo à organização de seguros e planos privados disponibilizados por grupos de profissionais de saúde 'liberais', o que se apresenta como uma das mais graves consequências das medidas neoliberais, comprometendo o potencial político-organizativo e a gestão desses trabalhadores (CARVALHO; SANTOS, CAMPOS, 2013).

No entanto, verificou-se que as tendências de precarização das relações e a flexibilização das condições de trabalho, ocorridas a partir dos anos 1990, introduzidas pelo Plano Diretor da Reforma do Estado, cujas leis decorrentes regulamentaram a utilização de instituições privadas em associação com o Estado (BRASIL, 1995), não se mantiveram nas mesmas proporções iniciais. Ou seja, mesmo com as dificuldades que os gestores possam ter encontrado, como a complexidade da organização de concursos públicos, a fixação de profissionais e a Lei de Responsabilidade Fiscal (BRASIL, 2000), que estipula limites para gastos com pessoal e outras regulamentações que regem a administração pública, estão viabilizando meios para diminuir a precarização da força de trabalho em saúde.

Tais resultados sinalizam avanços na gestão do trabalho pelos pequenos municípios, se comparados a dois estudos anteriores (TOMASI ET AL., 2008; GIRARDI; CARVALHO, 2001). O primeiro pesquisou, em 2005, trabalhadores da $A B$ na região Sul, em municípios com mais de 100.000 habitantes, e identificou que $59,0 \%$ destes haviam sido selecionados por concurso público, e 28,0\% admitidos por meio de vínculos precários. E o outro, em 2001, indicou que, entre as regiões geográficas do Brasil, a região Sul apresentava os menores índices de participação das prefeituras na contratação dos profissionais das EqSF, com os índices de $76,2 \%$ para os dentistas, $72,8 \%$ para os Aenf, $67,2 \%$ para os enfermeiros, $62,1 \%$ para os médicos e $53,6 \%$ para os ACS.

Um fator limitante, que ainda se apresenta nos pequenos municípios, é a quase ausência de uma política de planos de carreira, cargos e salários, pois $79,5 \%$ de todos os profissionais estudados referiram não ter PCCS. A implantação do PCCS/SUS, com perspectivas de desenvolvimento da carreira, política definida para ingresso, evolução e avaliação de desempenho, deve contribuir para maior valorização e fixação dos profissionais nas equipes, para maior satisfação e realização profissional; e, no âmbito da gestão, como facilitador para o dimensionamento das equipes, planejamento e previsão orçamentária, sendo de atribuição e responsabilidade dos gestores das três esferas de governo sua elaboração e implantação conforme 'Princípios e diretrizes para a gestão do trabalho no SUS' (BRASIL, 2005).

A implantação e a implementação do PCCS podem refletir, indiretamente, na melhoria da qualidade do trabalho e no atendimento à população, tendo em vista as especificidades das equipes e a complexidade do trabalho em saúde. O desenvolvimento de mecanismos mais eficazes, que contemplem tanto as exigências de qualidade nos serviços quanto os anseios de melhores condições de trabalho para os profissionais, é um desafio, ainda, a ser superado (PIERANTONI ET AL., 2008),

Quanto aos salários, chama atenção o desequilíbrio entre os salários pagos aos profissionais de nível superior. Aos médicos pagava-se, em média, mais que o triplo dos salários dos dentistas e enfermeiros. De qualquer forma, mesmo os maiores salários podem ser considerados 'baixos', pois, já em 2009, discutia-se no Senado Federal o Projeto de Lei do Senado n. ${ }^{\circ} 140$, que pretendia fixar o piso salarial para médicos e dentistas em sete mil reais por 20 horas semanais, bem acima dos valores salariais referidos pela maioria dos entrevistados dessas categorias (BRASIL, 2009). Esse fato 
provavelmente se relaciona com outros dois observados: a multiplicidade de vínculos empregatícios e as cargas horárias contratuais não cumpridas por médicos e dentistas. A adoção de PCCS, embora provavelmente implique maiores gastos, pode contribuir para resolver ou atenuar essas distorções.

\section{Considerações finais}

As informações apresentadas neste estudo propiciam um maior conhecimento das características da força de trabalho na AB em MPP.

A pesquisa revelou vários aspectos positivos, como a diversidade de categorias profissionais, a boa distribuição geral de trabalhadores de saúde por habitantes, a formação qualificada para atuação na $\mathrm{AB}$, a seleção por concurso ou teste seletivo, vínculos empregatícios formais e legais. Por outro lado, evidenciou deficiências a serem sanadas, como o número insuficiente de médicos para a população considerada, ausência de planos de carreira, salários insuficientes e, por vezes, iníquos entre trabalhadores, multiplicidade de vínculos empregatícios e descumprimento da carga horária contratada por alguns profissionais.

Problemas identificados podem ser superados pontual e especificamente, mas, assumindo uma visão mais abrangente, há necessidade da instituição de políticas específicas para os MPP. Concordando com o Plano Diretor em Municípios de Pequeno Porte, pode-se afirmar que são necessários investimentos técnicos, financeiros e de qualificação da gestão, bem como a realização de pesquisas, medidas de acompanhamento e avaliação por parte das instâncias estaduais e federal, devido à carência de estrutura para o planejamento e a baixa capacidade de gestão urbana para exercer a competência municipal constitucional dessa parcela de municípios. O apoio possibilitará a estes darem respostas às necessárias transformações e aos desafios contemporâneos da gestão do trabalho no SUS e suas especificidades, uma vez que a descentralização lhes trouxe desafios que ainda não foram superados completamente.

\section{Referências}

BRASIL. Lei Complementar $n^{\circ} 101$, de 04 de maio de 2000. Estabelece normas de finanças públicas voltadas para a responsabilidade na gestão fiscal e dá outras providências. Diário Oficial [da] União, Brasília, DF, 2000. Disponível em: <http://www.planalto.gov.br/ccivil_03/ leis/lcp/lcp101.htm>. Acesso em: 23 jun. 2013.

Ministério das Cidades. Plano Diretor em

Municípios de pequeno porte. [s.d.] . Disponível em: <http:// portal.cnm.org.br/sites/8800/8875/download/2_dia/ PlanoDiretoremMunicipiosdepequenoporte_Modificada. pdf>. Acesso em: 28 set. 2009.

Ministério da Saúde. Conselho Nacional de Saúde. Princípios e diretrizes para a gestão do trabalho no SUS (NOB/RH-SUS). 3 ed. Brasília: Ministério da Saúde, 2005.

\footnotetext{
. Ministério da Saúde. Secretaria de Atenção à Saúde. Departamento de Atenção Básica. Política Nacional de Atenção Básica. Brasília, DF: Ministério da Saúde, 2012. (Série E. Legislação em Saúde).

Ministério da Saúde. Secretaria de Gestão do Trabalho e da Educação na Saúde. Departamento de Gestão e da Regulação do Trabalho em Saúde. Comitê Nacional Interinstitucional de Desprecarização do Trabalho no SUS. Orientações gerais para elaboração de editais: processo seletivo público (agentes comunitários de saúde e agentes de combate às endemias). Brasília: Ministério da Saúde, 2006.
}

Ministério do Planejamento, Orçamento e Gestão. Plano diretor da reforma do Estado. Brasília. 
1995. Disponível em: <http://www.planejamento.gov. $\mathrm{br} /$ noticia. asp? $\mathrm{p}=$ not $\& \operatorname{cod}=524 \& \mathrm{cat}=238 \& \mathrm{sec}=25>$. Acesso em: 23 jun. 2013.

Senado Federal. Projeto de lei do senado $n^{\circ} 140$, de 2009. Altera dispositivos da Lei ${ }^{\circ} 3.999$, de 15 de dezembro de 1961, para fixar o valor do piso salarial e a jornada de trabalho dos médicos e cirurgiões-dentistas, e dá outras providências. Diário Oficial [da] União, Brasília, DF, 04 maio 2009. Disponível em: $<$ http://www.senado.gov.br/atividade/materia/getPDF. asp?t=59198\&tp=1>. Acesso em: 27 mar. 2014.

\section{CAMPOS, E. C.; MACHADO, M. H.; GIRARDI, S. N.} A fixação de profissionais de saúde em regiões de necessidades. Divulgação em Saúde para Debate, Rio de Janeiro, n. 44, p. 13-24, maio 2009.

CARVALHO, M.; SANTOS, N. R.; CAMPOS, G. W. S. A construção do SUS e o planejamento da força de trabalho em saúde no Brasil: breve trajetória histórica. Saúde em debate, Rio de Janeiro, v. 37, n. 98, p. 372-387, 2013.

GIRARDI, S. N.; CARVALHO, C. L. Contratação e qualidade do emprego no Programa de Saúde da Família no Brasil. 2001. Disponível em: < https://www.nescon. medicina.ufmg.br/pdf/\%5b2003\%5dcontracao_qualidade_emprego.pdf>. Acesso em: 23 jun. 2013.

\section{INSTITUTO BRASILEIRO DE GEOGRAFIA E} ESTATÍSTICA (IBGE). Censo. 2010. Disponível em: <http://www.ibge.gov.br/home/estatística/população/ censo2000>. Acesso em: 13 jul. 2010.

LACAZ, F. A. C. Relatório técnico final de projeto de pesquisa: avaliação da gestão do trabalho como tecnologia em saúde na atenção básica do Sistema Único de Saúde no Estado de São Paulo. São Paulo: Unifesp, 2008.

MENDONÇA, M. H. M. et al. Desafios para gestão do trabalho a partir de experiências exitosas de expansão da Estratégia Saúde da Família. Ciência $\mathcal{E}$ Saúde Coletiva, Rio de Janeiro, v. 15, n. 5, p. 2355-2365, 2010.
PARANÁ. Secretaria Estadual de Saúde. Plano Estadual de Saúde 2012-2015. Disponível em: <http://www.saude.pr.gov.br/arquivos/File/plano_estadual_saude_1104. pdf $>$. Acesso em: 27 mar 2014.

PIERANTONI, C. R. et al. Gestão do trabalho e da educação em saúde: recursos humanos em duas décadas do SUS. Physis - Revista de Saúde Coletiva, Rio de Janeiro. v. 18, n. 4, p. 685-704, 2008.

PINTO, I. C. M.; TEIXEIRA, C. F.; Formulação da política de Gestão do Trabalho e Educação na Saúde: o caso da Secretaria Estadual de Saúde da Bahia, Brasil, 2007-2008. Cad. Saúde Pública, Rio de Janeiro, v. 27, n. 9, p. 1777-1788, 2011.

RAMOS, A. S. et al. Política de Gestão do Trabalho e Educação Permanente na Bahia: "O SUS é uma escola". Revista Baiana de Saúde Pública. v. 33, n. 1, p. 40-50, jan./mar. 2009.

\section{SCHERER, M.D.A; PIRES, D.; SCHWARTZ, Y.}

Trabalho coletivo: um desafio para a gestão em saúde, Rev. Saúde Públ., São Paulo, v. 43, n. 4, p. 721-725, 2009.

TOMASI, E. et al. Perfil sócio-demográfico e epidemiológico dos trabalhadores da atenção básica à saúde nas regiões Sul e Nordeste do Brasil. Cad. Saúde Pública, Rio de Janeiro, v. 24, supl. 1, p. s193-s201, 2008.

WERMELINGER, M. et al. Feminilização do Mercado de Trabalho em Saúde no Brasil. Divulgação em Saúde para Debate, Rio de Janeiro, n. 45, p. 54-70, maio 2010.

\footnotetext{
Recebido para publicação em março de 2014

Versão final em julho de 2014

Conflito de interesses: inexistente

Suporte financeiro: Fundação Araucária, por meio da chamada de Projetos 08/2009 - Programa de Pesquisa para o SUS: Gestão

Compartilhada em Saúde. № do processo: 11348/2010
} 